\title{
Defect Dynamics in Graphene
}

\author{
Aalim M. Malik, National Institute of Technology, Srinagar, India \\ M. Ashraf Shah, National Institute of Technology, Srinagar, India \\ Nikhilesh K. Dilwaliya, National Institute of Technology, Srinagar, India \\ Vikash Dahiya, National Institute of Technology, Srinagar, India
}

\begin{abstract}
The experimental and theoretical study of graphene, two-dimensional (2D) graphite, is an extremely rapidly growing field of today's condensed matter research. Different types of disorder in graphene modify the Dirac equation leading to unusual spectroscopic and transport properties. The authors studied one of the disorders (i.e., grain boundaries) and formulated a theoretical model of graphene grain boundary by generalizing the two-dimensional graphene Dirac Hamiltonian model. In this model only, the authors considered the long-wavelength limit of the particle transport, which provides the main contribution to the graphene conductance. In this work, they derived the Hamiltonian in a rotated side dependent reference frame describing crystallographic axes mismatching at a grain boundary junction and showed that properties like energy spectrum are an independent reference frame. Also, they showed one of the topological property of graphene.
\end{abstract}

\section{KEYWORDS}

Dirac Equation, Disorders, Grain Boundaries, Graphene

\section{INTRODUCTION}

It is the second law of thermodynamics that dictates the presence of a certain amount of disorder in crystalline materials. But it is also due to the imperfection of material production processes that impurities and defects are always present in crystals. Such lattice imperfections have a strong influence on the electronic, optical, thermal, and mechanical properties of the solid. In fact, many of the characteristics of technologically important materials such as the conductance of semiconductors or the mechanical strength and ductility of metals are governed by defect (Kittel, 1953).Defects in bulk crystals have been studied extensively for many decades. Two dimensional crystals, however, have been considered only recently. Two graphite rods from two empty mosquito repellent refills were employed for synthesis of few-layered graphene for material developments on batteries and 
supercapacitor applications. The graphene sheet is isolated from graphite rod via electrochemical exfoliation method (Udhaya Sankar, 2018).

In fact, it was believed for a long time that they would be structurally unstable because of long wavelength fluctuations according to the Mermin Wagner theorem (Mermin, 1968). The situation changed, however, when single-layers of graphene were isolated for the first time by mechanical exfoliation (Novoselov, 2004). Graphene consists of a hexagonal monolayer network of sp2 - hybridized carbon atoms. Graphene and its structural counterpart, hexagonal boron nitride, are the only twodimensional crystalline materials we know today (Geim, 2009) (Britnell, 2012). The properties of graphene were expected to be outstanding, based on calculations addressing graphene as the parent material for carbon nanotubes. Therefore, the availability of graphene for experiments initiated a massive body of research, especially after large-scale synthesis methods like chemical vapor-deposition (Bae, 2010) and epitaxial growth (Kim, 2009) (Berger, 2004) on metal and SiC substrates were developed. Indeed, the predicted extraordinary properties have now been confirmed in many studies. Graphene is a single layer of graphite, the remarkable about it is that its crystalline structure is two dimensional. In other words the atoms in graphene are laid flat like billiard balls on a table. Just like in graphite each layer of graphene is made of hexagonal rings of carbon like lots of benzene rings connected together only with more carbon atoms replacing hydrogen atoms around the edge giving graphene a honeycomb structure. Some of these properties can only be observed at an extremely low defect concentration, which, as we discuss later, is possible because of the high formation energies of point defects in graphene. Nevertheless, like in any other real material, structural defects do exist in graphene and can dramatically alter its properties. Defects can also be deliberately introduced into this material, for example, by irradiation or chemical treatments which change graphene from crystalline to amporphous.

Graphene is a two-dimensional honeycomb lattice constituted by carbon atoms (Shah M. A and Shah K.A). Its reciprocal lattice determines a hexagonal Brillouin zone having six corners (K/K' points) where the low energy part of the bands structure is well described by a linear energymomentum dispersion relation, defining the so-called Dirac cone. The existence of Dirac cones in the graphene bands structure can be understood by using a tight-binding model. Consequently, the particle dynamics in graphene lattice follows the Dirac equation (Sarma, 2011), being the latter the manifestation of an emergent ultra relativistic behavior in a many-body system initially described by the Schr" odinger equation. Due to its unique band structure, in the past few years graphene has attracted much attention and intriguing transport properties, such as Klein tunneling (Allain, 2011), Zitterbewegung effect (Rusin, 2008), antilocalization (Tikhonenko, 2009), anomalous quantum Hall effect (Ostrovsky, 2008), Veselago focusing effect (Cheianov, 2007), have been suggested and, in some cases, experimentally proven. Apart from its theoretical interest, graphene is a two-dimensional chemical homogeneous system characterized by very high electrical mobility (Banszerus, 2015) and extraordinary mechanical properties (Papageorgiou, 2017), making it appealing in nanoelectronics and for flexible electronics implementations(Lee, 2015).

Crystals are never perfect and have various types of imperfections. These defects may affect many of their physical and mechanical properties, which in turn affect many important engineering properties. Graphene has been attracted a lot of interests as one of fashionable materials since it has opened horizons for physics exploration and future technology as a two dimensional material with extraordinary physical properties (Novoselov K. S., 2005) (Geim A. K., 2010). In general, the properties of graphene can be affected by structural irregularities such as dislocation, grain boundary, and edge structure (Han, 2007) (Son, 2006) so that those defect structures in graphene have been subjects of study. These imperfections are classified according to their geometry and shape. Of them the two dimensional defects that include external surfaces and grain boundaries have profound effect on electronic states of materials. The properties of polycrystalline materials are often dominated by the size of their grains and by the atomic structure of their grain boundaries. These effects should be especially pronounced in two dimensional materials, where even a line defect can divide and disrupt 
Figure 1. (a) Schematic of the region close to a grain boundary (GB region).Apart from the grainboundary region, where lattice distortions and vacancies affect honeycomb lattice structure ofgraphene, the bulk of the system is described by a regular atomic arrangement in which the latticeorientation is subject to a rotation of a definite angle going from the left to the right side of the junction. (b) The long-wavelength limit of the grain boundary junction depicted in panel. (b) The long-wavelength limit of the grain boundary junction depicted in panel.
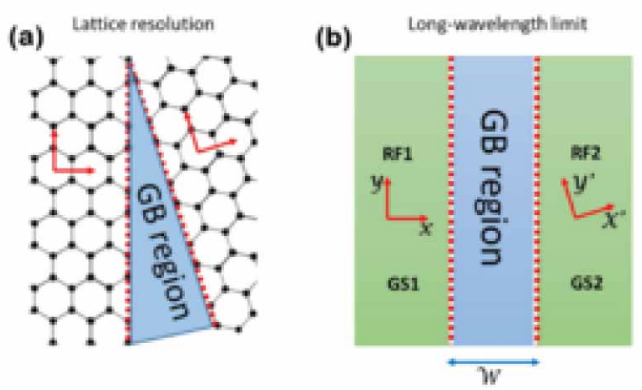

a crystal. Dislocations in carbon hexagonal lattice have been well documented from time to time and recently significant progress has been made to reveal the structures of grain boundaries and their influence on properties of graphene.

Graphene is always grown with different defects, among which the grain boundary (GB) is one of the most frequently formed defects (Duong, 2012). GBs provide numerous novel possibilities for modifying graphene such as tuning the charge distribution and transport properties. Therefore, GBs in graphene have been the focus of numerous studies due to their great significance in science and application (Liu, 2010). Intensive studies have been carried out to study the broad properties of GBs (Li, 2012). By analyzing the symmetry between the Brillouin zones of two sides, a theory was developed to predict the electronic transport properties through GBs (Yazyev, 2010) indicating that symmetric GBs have zero transport gap, but the asymmetric GBs have finite gaps. Such predictions are confirmed by nonequilibrium Green's function (NEGF) calculations (Zhang, 2012), and provide promising potential to regulate the electronic transport properties. As a result, some heterojunctions have already been designed using GBs to develop new transport devices (Li, 2011).

Researchers have developed some methods to construct GB structures, e.g., Yazyev and Louie used elementary topological defects to build dislocations and GBs in graphene (Yazyev and Louie, 2010). However, if we extend the 2D single composition GB to three dimensional (3D) multicomposition compound interfaces, the complexity of constructing structures from elementary defects would exponentially increase. One kind of promising method to predict complex structures is global optimization, which has been successfully applied to predict both 2D and 3D crystal structures (Trimarchi, 2007). However, even though this method is so powerful, it may still have a lot of difficulties to overcome in predicting the interface structure, which is usually much more complicated than the corresponding bulk systems, largely increasing the complexity of the task. Initial efforts towards interface structure prediction have been made in some preceding work using global optimization algorithms (von Alfthan, 2010).

\section{DIRAC HAMILTONIAN AND BOUNDARY CONDITIONS}

We will develop a theoretical model of graphene grain boundary by generalizing the graphene Dirac Hamiltonian model. Here we will use a continuous approach which is justified within the long wavelength limit assumed throughout this work. Using symmetry-based arguments, we derive unconventional boundary conditions characterizing the grain boundary physics and analyze their implications on the transport properties of the system. 
In the case of two space dimensions $(\mathrm{D}=2)$, which is of interest in graphene, we can write the Hamiltonian as:

$H^{2 D}=c s \cdot p+b m c^{2}$

where $s=\left(s^{x}, s^{y}\right)$ are Paulis matrices . As Electrons propagating through graphene's honeycomb lattice effectively lose their mass, producing quasi-particles that are described by a 2D analogue of the Dirac equation rather than the Schrodinger equation and move with fermi velocity $v_{F}$ rather that the velocity of light $\mathrm{c}$. Therefore putting $\mathrm{m}=0$ and replacing velocity of light with fermivelocity, the Hamiltonian in equation (1) for the description of the low-energy properties of electrons in graphene, can be written as:

$$
H_{\xi}=v_{F}\left(p_{x} \sigma^{x}+p_{y} \sigma^{y}\right)
$$

Now we will write the Dirac Hamiltonian of the graphene sheet GS2, in Fig.1, in terms of the reference frame of the graphene sheet GS1. The Hamiltonian in equation (2) for GS2 written in terms of RF2 takes the form:

$$
H_{\xi}=\xi v_{F}\left(p_{x} \sigma^{x}+p_{y} \sigma^{y}\right)
$$

We have introduced the valley isospin $x= \pm$, where $x=+$ denotes the $\vec{k}$ point at $+\mathrm{K}$ and $x=$ - the $\vec{k}$ point at $-\mathrm{K}$ called the Dirac points.

Using Pauli matrices The Hamiltonian in equation (3) changes into the form:

$$
\begin{aligned}
& H_{\xi}=\xi v_{F}\left[p_{x}\left(\begin{array}{ll}
0 & 1 \\
1 & 0
\end{array}\right)+p_{y}\left(\begin{array}{cc}
0 & -i \\
i & 0
\end{array}\right)\right] \\
& H_{\xi}=\xi v_{F}\left[\left(\begin{array}{cc}
0 & p_{x} \\
p_{x} & 0
\end{array}\right)+\left(\begin{array}{cc}
0 & -i p_{y} \\
i p_{y} & 0
\end{array}\right)\right] \\
& H_{\xi}=\xi v_{F}\left(\begin{array}{cc}
0 & p_{x}-i p_{y} \\
p_{x}+i p_{y} & 0
\end{array}\right)
\end{aligned}
$$

where $p_{x}=i \hbar \partial_{x}$ and $p_{y}=i h \partial_{y}$ are the quantum mechanical operators associated with the linear momentum components of quasiparticles. The matrix structure of the Hamiltonian originates from the presence of two atoms inside the unit cell of the graphene Bravais lattice. Accordingly the wave function $y(x, x)$ and $\left(y_{A}, y_{B}\right)^{T}$ describing the quantum state of the charge carriers is a two-component spinor whose components are related to the probability of finding the particle on the atom A or B of the unit cell.The correspondence between the wave functions expressed in RF1 or RF2 is established observing that thea-component of the wave function written in RF1,i.e $\phi_{\alpha}(x, y)$, is related to the homologous equation in RF2 by the equation $\phi_{\alpha}(x, y)=\phi_{\alpha}(x, y)$. The relation between distinct reference frames is simply given by a two dimensional rotation: 
$\left(\begin{array}{l}x^{\prime} \\ y^{\prime}\end{array}\right)=\left(\begin{array}{cc}\cos \theta & \sin \theta \\ -\sin \theta & \cos \theta\end{array}\right)\left(\begin{array}{l}x \\ y\end{array}\right)$

with $q$ an appropriate rotation angle. From the above observations one obtains useful relations linking the partial derivatives of the wave functions in the form:

$\left(\begin{array}{l}\partial_{x} \phi_{\alpha}(x, y) \\ \partial_{y} \phi_{\alpha}(x, y)\end{array}\right)=\left(\begin{array}{cc}\cos \theta & -\sin \theta \\ \sin \theta & \cos \theta\end{array}\right)\left(\begin{array}{c}\partial_{x^{\prime}} \phi_{\alpha}\left(x^{\prime}, y^{\prime}\right) \\ \partial_{y^{\prime}} \phi_{\alpha}\left(x^{\prime}, y^{\prime}\right)\end{array}\right)$

Using equation (3) in evaluating the quantity $H_{+} y\left(\mathrm{x}^{\prime}, \mathrm{y}^{\prime}\right)$, one easily get the equality:

$H_{+} y(x, y)=H_{q} \mathrm{~F}(x, y)$

where:

$H_{\theta}=v_{F}\left(\begin{array}{cc}0 & e^{i \theta}\left(p_{x}-i p_{y}\right) \\ e^{i \theta}\left(p_{x}+i p_{y}\right) & 0\end{array}\right)$

represents the Hamiltonian of GS2 written in terms of the RF1. Solving the eigenvalues problem $H_{q} y=$ Eywith usual planewave $=(\alpha, \beta)^{T} e^{i\left(k_{x} x+k_{y} y\right)}$, the eigenstates and the eigenvalues are: $\psi_{v}=\frac{1}{\sqrt{2}}\left(\begin{array}{c}1 \\ v e^{i(\phi-\theta)}\end{array}\right) e^{i \vec{k} \cdot \vec{r}}, E_{v}= \pm h v_{F}|k|(8)$

Here plus sign represents conduction band and minus valence band. $\vec{k}$ is the particle vector and $\vec{r}$ is the coordinate vector. The above results show that physical properties of the system, like e.g. the energy spectrum, are reference-frame independent and are clearly reminiscent of the rotational invariance of a bulk (monocristalline) graphene sheet. Also note that if the phase $q$ is rotated by $2 p$, the wave function changes sign indicating a phase of $p$. This is an example of a topological property, as it is insensitive to small deviations.

Setting the RF1 as a global reference frame, the Hamiltonian of the entire system $H_{g b}$ takes the piecewise form:

$H_{g b}=\left\{\begin{array}{c}H_{\theta \rightarrow 0}, x<0 \\ H_{\theta}, x>0\end{array}\right.$

The line $\mathrm{x}=0$ defines the grain boundary region in the limit $W \rightarrow 0$, which is an appropriate approximation when the grain boundary extension $W$ along the $\mathrm{x}$-direction is negligible. This requirement is always met by real grain boundaries which are defected regions where $W$ covers at least few lattice sites. 


\section{CONCLUSION}

We have proposed a continuous model in order to study effects of grain in graphene. Different types of disorder in graphene modify the Dirac equation leading to unusual spectroscopic and transport properties. We studied one the disorders i.e. Grain boundaries and formulate a theoretical model of graphene grain boundary by generalizing the two dimensional graphene Dirac Hamiltonian model. In this model only we considered the long-wavelength limit of the particle transport, which provides the main contribution to the graphene conductance. In this work we derived the Hamiltonian in a rotated side dependent reference frame describing crystallographic axes mismatching at a grain boundary junction and showed that one of the property like energy spectrumare reference-frame independent and are clearly reminiscent of the rotational invariance of a bulk (monocristalline) graphene sheet.

\section{ACKNOWLEDGMENT}

The authors are thankful to many colleagues and friends particularly Mir Faizal, Mateen Tantray, Arshid mir, Reyaz Shergojri, Jaffar Farooq and Mir Mudasir for their fruitful discussions while writing this article. The authors are also thankful to National Institute of Technology Srinagar and Nano-Mission for providing the required facilities in doing research work. 


\section{REFERENCES}

Allain, P. E., \& Fuchs, J. N. (2011). Klein tunneling in graphene: Optics with massless electrons. The European Physical Journal B, 83(3), 301-317.

Bae, S., Kim, H., Lee, Y., Xu, X., Park, J. S., Zheng, Y., \& Kim, Y. J. et al. (2010). Roll-to-roll production of 30-inch graphene films for transparent electrodes. Nature Nanotechnology, 5(8), 574.

Banszerus, L., Schmitz, M., Engels, S., Dauber, J., Oellers, M., Haupt, F., \& Stampfer, C. et al. (2015). Ultrahighmobility graphene devices from chemical vapor deposition on reusable copper. Science Advances, 1(6), e1500222.

Banszerus, L., Schmitz, M., Engels, S., Dauber, J., Oellers, M., Haupt, F., Watanabe, K., Taniguchi, T., Beschoten, B., \& Stampfer, C. (2015). Ultrahigh-mobility graphene devices from chemical vapor deposition onreusable copper. Science Advances, 1, e1500222-e1500222. doi:10.1126/sciadv.1500222

Berger, C., Song, Z., Li, T., Li, X., Ogbazghi, A. Y., Feng, R., \& De Heer, W. A. et al. (2004). Ultrathin epitaxial graphite: 2D electron gas properties and a route toward graphene-based nanoelectronics. The Journal of Physical Chemistry B, 108(52), 19912-19916.

Britnell, L., Gorbachev, R. V., Jalil, R., Belle, B. D., Schedin, F., Katsnelson, M. I., \& Castro Neto, A. H. et al. (2012). Electron tunneling through ultrathin boron nitride crystalline barriers. Nano Letters, 12(3), 1707-1710.

Bylinkin, A., Titova, E., Mikheev, V., Zhukova, E., Zhukov, S., Belyanchikov, M., \& Svintsov, D. et al. (2019). Tight-Binding Terahertz Plasmons in Chemical-Vapor-Deposited Graphene. Physical Review Applied, 11(5), 054017.

Cheianov, V. V., Fal'ko, V., \& Altshuler, B. L. (2007). Veselago lens for electrons: Focusing and caustics in graphenepn junctions. arXiv preprint cond-mat/0703410.

Di Bartolomeo, A., Giubileo, F., Romeo, F., Sabatino, P., Carapella, G., Iemmo, L., \& Lupina, G. et al. (2015). Graphene field effect transistors with niobium contacts and asymmetric transfer characteristics. Nanotechnology, 26(47), 475202.

Duong, D. L., Han, G. H., Lee, S. M., Gunes, F., Kim, E. S., Kim, S. T., \& Chae, S. J. et al. (2012). Probing graphene grain boundaries with optical microscopy. Nature, 490(7419), 235-239.

Geim, A. K. (2009). Graphene: status and prospects. Science, 324(5934), 1530-1534.

Geim, A. K., \& Novoselov, K. S. (2010). The rise of graphene. In Nanoscience and technology: a collection of reviews from nature journals (pp. 11-19). Academic Press.

Han, M. Y., Özyilmaz, B., Zhang, Y., \& Kim, P. (2007). Energy band-gap engineering of graphenenanoribbons. Physical Review Letters, 98(20), 206805.

Huang, M., Yan, H., Heinz, T. F., \& Hone, J. (2010). Probing strain-induced electronic structure change in graphene by Raman spectroscopy. Nano Letters, 10(10), 4074-4079.

Huang, P. Y., Ruiz-Vargas, C. S., Van Der Zande, A. M., Whitney, W. S., Levendorf, M. P., Kevek, J. W., \& Park, J. et al. (2011). Grains and grain boundaries in single-layer graphene atomic patchwork quilts. Nature, 469(7330), 389-392.

Jauregui, L. A., Cao, H., Wu, W., Yu, Q., \& Chen, Y. P. (2011). Electronic properties of grains and grain boundaries in graphene grown by chemical vapor deposition. Solid State Communications, 151(16), 1100-1104.

Kim, K. (2012). Structural Characterization, Manipulation, and Properties of Graphene Membranes (Doctoral dissertation). UC Berkeley.

Kim, K. S., Zhao, Y., Jang, H., Lee, S. Y., Kim, J. M., Kim, K. S., ... Hong, B. H. (2009). Large-scale pattern growth of graphene films for stretchable transparent electrodes. Nature, 457(7230), 706-710.

Kittel, C. (1953). Introduction to Solid State Physics. Academic Press.

Lee, S. M., Kim, J. H., \& Ahn, J. H. (2015). Graphene as a flexible electronic material: Mechanical limitations by defect formation and efforts to overcome. Materials Today, 18(6), 336-344. 
Li, X. F., Wang, L. L., Chen, K. Q., \& Luo, Y. (2011). Design of graphene-nanoribbonheterojunctions from first principles. The Journal of Physical Chemistry C, 115(25), 12616-12624.

Li, X. F., Wang, L. L., Chen, K. Q., \& Luo, Y. (2012). Electronic transport through zigzag/armchair graphenenanoribbonheterojunctions. Journal of Physics Condensed Matter, 24(9), 095801.

Liu, T. H., Gajewski, G., Pao, C. W., \& Chang, C. C. (2011). Structure, energy, and structural transformations of graphene grain boundaries from atomistic simulations. Carbon, 49(7), 2306-2317.

Liu, Y., \& Yakobson, B. I. (2010). Cones, pringles, and grain boundary landscapes in graphene topology. Nano Letters, 10(6), 2178-2183.

Mermin, N. D. (1968). Crystalline order in two dimensions. Physical Review, 176(1), 250.

Meyer, J. C., Chuvilin, A., Algara-Siller, G., Biskupek, J., \& Kaiser, U. (2009). Selective sputtering and atomic resolution imaging of atomically thin boron nitride membranes. Nano Letters, 9(7), 2683-2689.

Najar, K., Sheikh, N., Din, S., \& Shah, M. (2016). Effect of CVD-diamond coatings on the tribological performance of cemented tungsten carbide substrates. Academic Press.

Neto, A. C., Guinea, F., Peres, N. M., Novoselov, K. S., \& Geim, A. K. (2009). The electronic properties of graphene. Reviews of Modern Physics, 81(1), 109.

Novoselov, K. S., Geim, A. K., Morozov, S. V., Jiang, D., Katsnelson, M. I., Grigorieva, I., ... Firsov, A. A. (2005). Two-dimensional gas of massless Dirac fermions in graphene. Nature, 438(7065), 197-200.

Novoselov, K. S., Geim, A. K., Morozov, S. V., Jiang, D., Zhang, Y., Dubonos, S. V., ... Firsov, A. A. (2004). Electric field effect in atomically thin carbon films. Science, 306(5696), 666-669.

Ostrovsky, P. M., Gornyi, I. V., \& Mirlin, A. D. (2008). Theory of anomalous quantum Hall effects in graphene. Physical Review. B, 77(19), 195430.

Papageorgiou, D. G., Kinloch, I. A., \& Young, R. J. (2017). Mechanical properties of graphene and graphenebased nanocomposites. Progress in Materials Science, 90, 75-127.

Rusin, T. M., \& Zawadzki, W. (2008). Zitterbewegung of electrons in graphene in a magnetic field. Physical Review. B, 78(12), 125419.

Sarma, S. D., Adam, S., Hwang, E. H., \& Rossi, E. (2011). Electronic transport in two-dimensional graphene. Reviews of Modern Physics, 83(2), 407.

Shah, M. A., \& Shah, K. A. (2019). Science of Small-Nanotechnology. Wiley Pvt. Limited.

Son, Y. W., Cohen, M. L., \& Louie, S. G. (2006). Energy gaps in graphenenanoribbons. Physical Review Letters, 97(21), 216803.

Srivatsa, T. V. (2017). Graphene based surface coatings on ceramic membranes for water de-salination (Doctoral dissertation). Christian AlbrechtsUniversitätzu Kiel.

Tikhonenko, F. V., Kozikov, A. A., Savchenko, A. K., \& Gorbachev, R. V. (2009). Transition between electron localization and antilocalization in graphene. Physical Review Letters, 103(22), 226801.

Trimarchi, G., \& Zunger, A. (2007). Global space-group optimization problem: Finding the stablest crystal structure without constraints. Physical Review. B, 75(10), 104113.

Udhaya Sankar, G., Ganesa Moorthy, C., \& RajKumar, G. (2018). Synthesizing graphene from waste mosquito repellent graphite rod by using electrochemical exfoliation for battery/supercapacitor applications. Energy Sources. Part A, Recovery, Utilization, and Environmental Effects, 40(10), 1209-1214. doi:10.1080/1556703 6.2018 .1476609

von Alfthan, S., Benedek, N. A., Chen, L., Chua, A., Cockayne, D., Dudeck, K. J., \& Rühle, M. et al. (2010). The structure of grain boundaries in strontium titanate: Theory, simulation, and electron microscopy. Annual Review of Materials Research, 40, 557-599.

Yazyev, O. V., \& Louie, S. G. (2010). Electronic transport in polycrystalline graphene. Nature Materials, 9(10), 806-809. 
Yazyev, O. V., \& Louie, S. G. (2010). Topological defects in graphene: Dislocations and grain boundaries. Physical Review. B, 81(19), 195420.

Zhang, J., Gao, J., Liu, L., \& Zhao, J. (2012). Electronic and transport gaps of graphene opened by grain boundaries. Journal of Applied Physics, 112(5), 053713.

Zribi, B., Roy, E., Pallandre, A., Chebil, S., Koubaa, M., Mejri, N., \& Haghiri-Gosnet, A. M. et al. (2016). A microfluidic electrochemical biosensor based on multiwall carbon nanotube/ferrocene for genomic DNA detection of Mycobacterium tuberculosis in clinical isolates. Biomicrofluidics, 10(1), 014115. 